\title{
EPIDEMIOLOGIA DA DENGUE EM SÃO LUÍS - MARANHÃO, BRASIL, 2000 A 2007
}

\author{
EPIDEMIOLOGY OF DENGUE FEVER IN SÃO LUÍS - MARANHÃO, \\ BRAZIL, FROM 2000 TO 2007
}

\author{
EPIDEMIOLOGÍA DE DENGUE EN SÃO LUÍS - MARANHÃO, \\ BRASIL, 2000 A 2007
}

\author{
Darci Ramos Fernandes \\ Edenilde Alves dos Santos \\ Adely Fátima Dutra Vieira Araújo \\ Claudio Zannoni \\ Ana Hélia de Lima Sardinha \\ Zulimar Márita Ribeiro Rodrigues
}

\begin{abstract}
Resumo: Nas últimas décadas as epidemias de dengue têm sido responsáveis por um número elevado de casos de mortalidade no mundo, tornando-se um grave problema de saúde pública. Países de climas tropicais são mais afetados devido às condições ambientais, climáticas e sociais, tendo o clima como fator importante na distribuição temporal e espacial de doenças transmitidas por vetores, especificamente a dengue. Este estudo epidemiológico, observacional, descritivo e seccional objetiva descrever as características epidemiológicas da dengue em São Luís - Maranhão, Brasil, no período de 2000 a 2007. Foram analisados 10.792 casos registrados no período pelo Sistema de Informação de Arquivos de Notificação (SINAN). Verificou-se que a prevalência da dengue apresentou constante variação sendo que a maior incidência foi no sexo feminino e os mais acometidos foram indivíduos na faixa etária de 20 a 34 anos. A análise revelou um perfil endêmico com picos que merecem atenção e esforço das autoridades sanitárias e da população em geral para a solução do problema.
\end{abstract}

Palavras-chave: Dengue. Epidemiologia. Vigilância epidemiológica.

Abstract: In recent decades dengue fever epidemics have been responsible for a high number of mortalities in the world, becoming a serious public health problem. Countries with tropical climates are more affected due to environmental, social and climate conditions, taking the weather as an important factor in the temporal and spatial distribution of vector-borne diseases, especially dengue fever. This is an epidemiological, observational and cross-sectional study aimed to describe the epidemiological characteristics of dengue fever in São Luís - Maranhão, Brazil, in the period of 2000 to 2007. Secondary data from the Information System of Notification Files (SINAN) were analyzed. 10,792 cases recorded in the period in Information System Files Notification (SINAN) were analyzed. It was found that the prevalence of dengue fever presented constant variation being the higher incidence in females and the most affected were individuals aged 2034 years. The analysis revealed an endemic profile with peaks that deserve attention and efforts of health authorities and the general population for the solution of the problem.

Keywords: Dengue fever. Epidemiology. Epidemiological supervision.

Resumen: En las últimas décadas, las epidemias de dengue han sido responsables de un elevado número de muertes en el mundo, convirtiéndose en un grave problema de salud pública. Los países tropicales se ven más afectados por las condiciones ambientales y climáticas teniendo el clima como un factor importante en la distribución temporal y espacial de las enfermedades transmitidas por vectores, especialmente del dengue. Se trata de un estudio epidemiológico, observacional descriptivo y seccional; y tiene como objetivo el describir las características epidemiológicas del dengue en São Luís - Maranhão, Brasil, en el período 2000-2007. Se analizaron 10.792 casos registrados en el período en el Sistema de Información de Archivos de Notificación (SINAN). Se encontró que la prevalencia de dengue presenta constantes variaciones es que la incidencia fue mayor en las mujeres y los más afectados fueron las personas de entre 20-34 años. El análisis reveló un perfil endémico con picos que merecen la atención y los esfuerzos de las autoridades sanitarias y la población en general para la solución del problema.

Palabras clave: Dengue. Epidemiología. Vigilancia epidemiológica.

\footnotetext{
*Artigo recebido maio 2013

Aprovado em junho 2013
} 


\section{INTRODUÇÃO}

A dengue é uma doença infecciosa transmitida por um vírus de genoma RNA que se caracteriza por apresentar quadro febril inespecífico, classificado clinicamente como febre do dengue ou dengue clássica, até manifestações graves com hemorragia, na febre hemorrágica do dengue e síndrome do choque do dengue, podendo levar a óbito. São conhecidos quatro sorotipos: DEN-1, DEN-2, DEN-3 e DEN-4. A dengue apresenta grande repercussão econômica e social, pois afeta a força de trabalho, prejudica o comparecimento escolar e a organização do atendimento à saúde (BRAGA; VALLE, 2007; DUARTE; FRANÇA, 2006; TORRES, 2005).

Independentemente do sexo ou idade, atinge todos os grupos sociais, afetando em maior índice aqueles que estejam mais susceptíveis ao vetor. A grande incidência de casos de dengue coincide com as ocorrências das chuvas, aumento da umidade relativa do ar e fatores contribuintes para a proliferação do vetor (GONÇALVES NETO; REBÉLO, 2004).

A transmissão da doença dá-se através da via mosquito-homem, que durante a fase larvária se prolifera nos reservatórios de água parada ou limpa, constituindo os criadouros do mosquito. Entre 10 a 14 dias a fêmea do Aedes aegypti contaminada infecta o indivíduo, sendo capaz de disseminar a doença por toda a vida da fêmea do mosquito. Aparece principalmente em países tropicais, onde as condições do ambiente favorecem a reprodução do mosquito vetor. O ser humano, além de ser a fonte de infecção, é também o reservatório vertebrado, fato este que torna necessário cada cidadão influir ativamente na promoção e manutenção de sua saúde, evitando mortes desnecessárias (BRASIL, 1996).

Em termos de morbidade e mortalidade, a dengue é considerada a mais importante virose transmitida por artrópodes que afeta o homem (FOCACCIA, 2006). Dessa forma, a grande expansão da disseminação por dengue tem atingido parcelas significativas da população brasileira, apresentando avanços significativos de óbitos pela doença.

No Brasil, os primeiros relatos sobre uma epidemia dessa doença datam de 1845, com casos no Rio de Janeiro. Ainda nesse século foram registradas duas epidemias de dengue: uma entre 1846-1848 e outra no período de 1851-1853. Dessa época até 1981 foram registradas apenas duas epidemias: uma em 1916 e outra em 1923. A partir de 1996, a dengue tornou-se endêmica em quase todos os estados brasileiros (BRASIL, 1996).

Segundo o Programa Nacional de Controle da Dengue, foram notificados 85.018 casos de dengue no país de 01 de janeiro até 12 de março de 2007 (BRASIL, 2007). Dados oficiais do Ministério da Saúde, publicados e acessíveis no site portal.saude.gov.br, relatam que no período de janeiro a fevereiro foram no tificados 79.732 casos da doença, o que representa um aumento de 29,58\% comparado ao mesmo período de 2006. Esse quadro demonstra uma circulação simultânea do vírus da dengue, tornando a população mais vulnerável às epidemias por dengue hemorrágica, o que pode desencadear cada vez mais casos de mortalidade.

$\mathrm{Na}$ região nordeste foram notificados, nesse mesmo período, 12.139 casos da dengue hemorrágica que comparados aos dados de 2006 apresentam um aumento de 1,1\%. No Maranhão, na capital São Luís, apenas no Hospital Universitário Materno Infantil, no período de fevereiro a março de 2007, foram notificados 53 casos suspeitos de dengue hemorrágica em crianças internadas. O número correspondia a $40 \%$ do total de internações de crianças com dengue hemorrágica em 2006 (BRASIL, 2007).

A reprodução da doença está intimamente relacionada aos determinantes de ordem socioeconômica, uma vez que a dengue pode ser considerada um subproduto da urbanização acelerada, sem planejamento, característica dos centros urbanos de países em desenvolvimento. Outros determinantes da doença são as migrações, viagens aéreas, deterioração dos sistemas de saúde, inexistência de vacina ou tratamento etiológico, grande fluxo populacional entre localidades e altos índices pluviométricos e de infestação pelo vetor (MACIEL; SIQUEIRA JR.; MARTELLI, 2008).

A cidade de São Luís apresenta características propícias para ser uma zona endêmica da dengue por possuir fatores climáticos compatíveis, grandes armazenamentos de água inadequados e baixa conscientização populacional para a importância de não contrair dengue. Também apresenta forte propensão para as contínuas reincidências de casos por dengue, principalmente no período chuvoso onde há maior probabilidade para o desenvolvimento da doença, devido à existência de criadouros domésticos e às barreiras sociais que impossibilitam a atuação intra e peri domiciliar dos agentes de saúde no controle ao mosquito, somando-se a necessidade de ações governamentais competentes para uma nova abordagem de combate ao Aedes aegypt além da falta de agilidade na realização dos exames por parte dos órgãos competentes (ARAÚJO; NUNES, 2005).

\subsection{Aspectos epidemiológicos}

A dengue é uma arbovirose transmitida ao homem pela picada do mosquito Aedes aegypti. Em âmbito mundial sua prevalência cresceu dramaticamente nas últimas décadas, quando a doença tornou-se endêmica em países da África, das Américas, da Região Leste do Mediterrâneo, do Sudeste Asiático e do Pacífico Ocidental. A Organização Mundial de Saúde (OMS) calcula que $40 \%$ da população mundial, cerca de 2,5 bilhões de pessoas vivendo em 
áreas tropicais e subtropicais estão em risco (SINGHI; KISSOON; BANSAL, 2007).

Segundo Singhi, Kissoon e Bansal (2007), todos os anos ocorrem cerca de 50 a 100 milhões de casos de dengue, 500.000 casos de dengue hemorrágica e, no mínimo, 12.000 mortes são relatadas no mundo. Noventa por cento dos óbitos ocorrem em crianças com menos de 15 anos.

No Brasil, desde 1986 ocorrem epidemias anuais de dengue, até então pelos sorotipos 1 e 2 , tornando-se um problema nacional de saúde pública (DONALÍSIO; GLASSER, 2002). No Rio de Janeiro, em 2002, ocorreu a maior epidemia de dengue já registrada com a introdução de um novo sorotipo (sorotipo 3), isolado pela primeira vez no estado em janeiro de 2001. Segundo Vabo et al. (2004), a introdução de um novo sorotipo em uma população suscetível propiciou o aparecimento de formas mais graves da doença. Nos últimos anos o número de casos registrados e de mortes vítimas da dengue aumentou, visto que o mosquito se tornou resistente aos inseticidas convencionais (HEMINGWAY et al., 2000 apud COSTA et al., 2005).

Segundo Gonçalves Neto e Rebêlo (2004), entre os anos de 1982 e 1997, a Região Nordeste contribuiu com 502.772 casos de dengue, ou $54,00 \%$ das notificações ocorridas no país; no Maranhão foram notificados, no mesmo período, 14.190 casos representando $2,83 \%$ dos casos nordestinos. No período decorrido entre 1998 e 2001 os casos nordestinos aumentaram para 652.448 registros, correspondendo a $46,30 \%$ dos casos nacionais, enquanto o Maranhão nesse mesmo período notificou 27.884 casos, ou $4,30 \%$ das notificações do Nordeste. Em 1996 aconteceu a primeira epidemia na ilha de São Luís com 4.641 casos notificados.

Para muitos estudiosos existe uma relevante associação entre a incidência da dengue e as estações chuvosas, as altas temperaturas, as altitudes e os ventos. De acordo com Donalísio e Glasser (2002), as epidemias no Sudeste Asiático, México, Brasil e Caribe, nas décadas de 1980 e 90, foram registradas em estações chuvosas, o que leva a consideração de que nesse período há maior possibilidade de propagação da doença.

A existência da dengue no Brasil é conhecida desde o século XVII, apresentando incidências em várias regiões do país. O Rio de Janeiro foi o estado onde ocorreram mais casos da epidemia de dengue, que na forma da febre amarela urbana já foi controlada e não ocorre no Brasil desde 1942. A vacinação da febre amarela é possível e indispensável a todas as pessoas que residem em áreas onde circula o vírus da febre amarela, ou viajem para esses locais (COSTA et al., 2005). Conforme Teixeira et al. (2002), há muitas décadas o Brasil vem desenvolvendo ações de combate ao Aedes aegypti, tendo, inclusive, alcançado sua erradicação em 1958, ficando livre deste vetor durante alguns anos. No entanto, após a sua reintrodução em 1976 não mais conseguiu eliminá-lo e, dez anos depois, a estratégia de erradicação foi substituída pelo controle, de onde têm sido criados diversos programas sem, contudo, alcançarem êxito total.

Em dezembro de 2001 ocorreu a maior e mais grave epidemia da dengue provocada pela introdução do sorotipo 3 do vírus da doença no país, tendo sido notificados mais de 1,2 milhão de casos em 2001 e 2002, ocorrendo também a co-circulação dos sorotipos 1 e 2. Após esse período, o processo endêmico-epidêmico manteve-se predominantemente nas grandes regiões metropolitanas do país, contribuindo também para a disseminação da doença para outros municípios. Isto acontece devido diversas características das regiões, como o processo de urbanização desordenado (MEDRONHO, 2006).

Segundo Toledo et al. (2006), dentre as doenças de notificação compulsória, a dengue é uma das que representa grave problema de saúde pública, sendo a arbovirose mais distribuída entre os seres humanos. Para Lima et al. (2007), a dengue tem se apresentado como importante problema de saúde pública no Brasil, onde a tendência de ocorrência em suas formas graves é crescente, apesar da oscilação dos níveis de transmissão anuais ao longo das últimas décadas. A esse respeito, Chiaravalloti Neto (2007) consideram a dengue uma das doenças infecciosas de maior incidência nas regiões intertropicais, e um subproduto da urbanização desordenada que se verifica em países de economia emergente. Seu vetor, o Aedes aegypti, apresenta grande adaptação à vida urbana e sua propagação é privilegiada devido aos hábitos consumistas modernos. Assim, na cadeia epidemiológica da dengue o único elemento controlável na atualidade é o seu vetor, sendo necessário o incremento de pesquisas para o estudo mais detalhado do comportamento do Aedes aegypti, para que assim medidas mais eficazes possam ser desenvolvidas no combate à sua proliferação.

No Brasil, há muitas décadas é realizado um programas de combate ao mosquito, entretanto, observa-se desde o início dos anos oitenta uma expansão geográfica da infestação do seu território e circulação progressiva e intensa deste vírus, com registro de grandes epidemias e de transmissão endêmica em diferentes centros urbanos (TEIXEIRA et al., 2002).

Em São Luís-MA o Aedes aegypti foi introduzido em 1969, mas só chamou a atenção dos órgãos de saúde no ano de 1995, quando se detectaram os primeiros casos de dengue clássica no bairro da Cohab-Anil. Por iniciativa do Ministério da Saúde, diante da gravidade da situação, foi montada uma estratégia de combate ao vetor com base no Plano Diretor de Erradicação do Aedes aegypti. Mediante convênio firmado entre o Ministério da Saúde e a prefeitura de São Luís, garantiu-se a im- 
plementação das atividades direcionadas ao combate do Aedes aegypti, que vêm se desenvolvendo desde o ano de 1995, sem que se obtenham resultados satisfatórios constantes (SÃO LUÍS, 2001).

De acordo com dados da Secretaria Municipal de Saúde de São Luís, a primeira epidemia de dengue no local aconteceu em 1996 com 4.641 casos notificados (SÃO LUÍS, 2003). Naquele ano realizou-se um inquérito soro epidemiológico estimando-se que $41,40 \%$ da população estudada se encontrava sensibilizada pelo DEN-1. A epidemia prosseguiu até 1998, havendo dificuldade para confirmação dos casos em laboratório, porém em 2001 foi isolado o sorotipo DEN-2 (GONÇALVES NETO; REBÊLO, 2004).

Diante da situação da dengue, fazem-se necessário estudos que demonstrem o panorama da prevalência da dengue, bem como os aspectos epidemiológicos envolvidos, a fim de propiciar uma importante fonte para os profissionais de saúde e, ao mesmo tempo, incentivar a população a desenvolver atividades de prevenção que contribuam para a redução de morbimortalidade por dengue. Pelo exposto, o presente artigo tem por objetivo descrever as características epidemiológicas do dengue em São Luís, Maranhão, no período de 2000 a 2007.

\section{MATERIAL E MÉTODOS}

Trata-se de um estudo epidemiológico observacional descritivo e seccional, o qual teve como objeto de estudo todos os casos de dengue investigados pela Secretaria Estadual de Saúde (SES) e utilizaram-se dados secundários registrados nos bancos de dados do Sistema de Informação de Agravos de Notificação (SINAN), bem como nas planilhas paralelas de dengue. Estas planilhas reúnem casos e óbitos que preenchem a definição de caso de febre hemorrágica do dengue, repassados pelas SES semanalmente, de forma paralela às notificações oficiais, para a Coordenação do Programa Nacional de Controle do Dengue do Ministério da Saúde, relativos aos aspectos epidemiológicos e da ocorrência e distribuição dos casos de dengue, no período epidêmico entre os anos de 2000 e 2007, no município de São Luís - MA (Brasil).

A Região Metropolitana de São Luís, também conhecida como Grande São Luís, encontra-se dividida em sete distritos sanitários: Centro, Itaqui-Bacanga, Coroadinho, Cohab, Bequimão, Tirirical e Vila Esperança. Sendo composta pelos municípios de São José de Ribamar, Raposa, Paço do Lumiar, Alcântara, Bacabeira e São Luís, situados no Estado do Maranhão. Juntos, perfazem uma população de 1.342.846 habitantes (IBGE, 2011). Situada no arquipélago de ilhas do Golfão Maranhense com mais de $1000 \mathrm{~km}^{2}$, dividida pelos rios Anil e Bacanga. Na região compreendida entre ambos os rios está o núcleo inicial de povoa- ção da região; a norte e oeste do rio anil ficam os bairros mais modernos e bem equipados da cidade, ao sul do bacanga está o distrito industrial da ilha. Nas zonas centro e norte da região metropolitana estão os grandes bairros populares, quase sempre conjuntos habitacionais, construídos até meados da década de oitenta. Nos extremos norte e leste a ocupação é esparsa; são áreas de praias desertas e manguezais fechados. No lado oeste da ilha ficam os bairros mais prósperos, as praias mais frequentadas e melhor infraestrutura. As avenidas Litorânea e dos Holandeses ligam os bairros da zona oeste entre si e à zona norte onde fica o município de Raposa. A avenida Jerônimo de Albuquerque formou um eixo de expansão para onde foi levado o desenvolvimento urbano da cidade, liga a área do São Francisco-Renascença à Cohab; em suas margens surgiram vários bairros residenciais e estabelecimentos comerciais. Os outros dois eixos importantes são os formados pela avenida Guajajaras ligando a zona sul às oeste e norte, e a estrada de Ribamar, que liga o centro da ilha aos bairros da região norte e às sedes dos municípios de Paço do Lumiar, São José de Ribamar e Raposa. O clima é o tropical quente e úmido, com duas estações: a chuvosa (janeiro a junho), com precipitação pluviométrica média de $1.954 \mathrm{~mm}$, e a de estiagem (julho a dezembro). A temperatura varia entre $28^{\circ} \mathrm{C}$ e $30^{\circ} \mathrm{C}$.

Para a coleta de dados, utilizou-se um formulário no qual constavam informações sobre os casos notificados referentes à idade, sexo, número de casos/ano e logradouro. As variáveis contidas no banco de dados escolhidas para análise foram: (a) temporal (ano); (b) demográficas (sexo e faixa etária).

O processamento dos dados ocorreu com auxílio de softwares, sendo que o programa estatístico JMP Statistics and Graphis Guide versão 3.2.6 foi utilizado para a entrada e tratamento dos dados, enquanto o Microsoft Office Excel 2007, por imprimir mais qualidade, foi utilizado na confecção de gráficos e tabelas. A análise estatística dos dados foi efetuada com base em frequências absolutas e relativas por períodos.

\section{RESULTADOS E DISCUSSÃO}

No quadro 1 , verifica-se que a prevalência da dengue no município de São Luís no período de 2000 a 2007 apresentou constante variação, estando em acentuada inclinação para o crescimento entre os anos de 2000 a 2003. No ano de 2004 apresentou uma baixa significativa nos casos notificados diminuindo as ocorrências em $50 \%$. No entanto no ano seguinte, ou seja, em 2005, os casos notificados da dengue apresentaram um aumento 4 vezes maior que no ano anterior. Embora em 2006 tivesse havido uma diminuição nas ocorrências ainda apresentou um índice elevado de notificações, entretanto, em 2007, o número de 
Quadro 1 - Distribuição de notificação de casos de dengue segundo o sexo, de acordo com SINAN no período de 2000 a 2007, São Luís - MA

\begin{tabular}{|c|c|c|c|c|c|c|}
\hline Ano & Total & masc & $\%$ & fem & $\%$ & $\%$ \\
\hline 2000 & 241 & 122 & 50,6 & 119 & 49,4 & 100 \\
\hline 2001 & 605 & 278 & 46 & 327 & 54 & 100 \\
\hline 2002 & 902 & 404 & 44,8 & 498 & 55,2 & 100 \\
\hline 2003 & 1059 & 507 & 48 & 552 & 52 & 100 \\
\hline 2004 & 526 & 241 & 46 & 285 & 54 & 100 \\
\hline 2005 & 2689 & 1188 & 44 & 1501 & 56 & 100 \\
\hline 2006 & 1338 & 680 & 51 & 658 & 49 & 100 \\
\hline 2007 & 3432 & 1613 & 47 & 1819 & 53 & 100 \\
\hline
\end{tabular}

Fonte: Elaborado pelos autores

notificações apresentou um aumento de 2,56 vezes em relação ao ano anterior.

O sexo de maior incidência da dengue nos casos notificados foi o sexo feminino em seis anos do período estudado, compreendendo os anos de 2001 a 2005 e 2007. Nos anos de 2000 e 2006 a prevalência de notificações foi para o sexo masculino, todavia a variação foi bastante pequena com o percentual de $1 \%$ de diferenciação.

Na Tabela 1, verifica-se que a faixa etária de maior incidência da dengue no período estudado foi a correspondente aos 20 a 34 anos de idade, compreendendo a população adulta de São Luís, também nas faixas etárias correspondentes à adolescência (10 a 14 anos e 15 a 19 anos) houve significativa prevalência de casos notificados.

Este estudo limitou-se à coleta de informações nos dados estatísticos do Sistema Nacio- nal de Agravos de Notificações. Sendo assim, deve-se considerar a existência de uma possível subnotificação, pois algumas variáveis não se encontravam registradas nos dados, bem como os fatores que contribuem para as infecções e avanço da dengue no município. Os resultados deste estudo demonstraram que a situação da dengue em São Luís no período em estudo foi preocupante uma vez que anualmente constatava-se o aumento do número de casos de notificações da doença. Este resultado se coaduna com o encontrado por Câmara et al. (2007) em estudo retrospectivo histórico da dengue no Brasil, onde foram consideradas as características regionais e dinâmicas das notificações da dengue e verificou-se que as regiões Nordeste e Sudeste detiveram cerca de $86 \%$ das notificações, enquanto as regiões Sul, Centro-Oeste e Norte são responsáveis por um número significativamente menor.

$\mathrm{Na}$ avaliação dos aspectos epidemiológicos da dengue no município de São Luís, Maranhão, no período de 1997 a 2002, Gonçalves Neto e Rebêlo (2004), constataram que a doença apresenta um padrão sazonal, com maior incidência de casos na estação chuvosa. Quanto às iniciativas voltadas ao controle da doença é inegável que haja uma ação da sociedade de todos os segmentos para que a dengue venha a ser erradicada. Chiaravalloti Neto (2007), em estudo sobre o controle da dengue em uma área urbana do Brasil, avaliaram os resultados da integração do Programa Saúde da Família (PSF) e do Programa de Controle do Dengue em São José do Rio Preto, São Paulo, Brasil. O estudo foi realizado em uma área com PSF e outra sem PSF. Os resultados

Tabela 1 - Distribuição de notificação de casos de dengue segundo faixa etária, de acordo com SINAN no período de 2000 a 2007, em São Luís - MA.

\begin{tabular}{|c|c|c|c|c|c|c|c|c|c|c|c|c|c|c|c|c|}
\hline Idades & 2000 & $\%$ & 2001 & $\%$ & 2002 & $\%$ & 2003 & $\%$ & 2004 & $\%$ & 2005 & $\%$ & 2006 & $\%$ & 2007 & $\%$ \\
\hline K ano & 0 & & 13 & 2,1 & 48 & 5,32 & 27 & 2,54 & 13 & 2,47 & 42 & 1,56 & 45 & 3,36 & 182 & 5,3 \\
\hline $1 \mathrm{a} 04$ & 3 & 1,24 & 32 & 5,21 & 56 & 6,2 & 43 & 4,06 & 12 & 2,28 & 68 & 2,52 & 137 & 10,23 & 409 & 11,91 \\
\hline $\mathrm{a} 09$ & 8 & 3,31 & 58 & 9,41 & 97 & 10,75 & 64 & 6,04 & 22 & 4,18 & 169 & 6,28 & 188 & 14,05 & 790 & 23,01 \\
\hline a 14 & 17 & 7,05 & 78 & 13 & 103 & 11 & 88 & 8,3 & 30 & 5,7 & 202 & 7,51 & 100 & 7,47 & 346 &, 08 \\
\hline 5 a 19 & 37 & 15,35 & 78 & 13 & 101 & 11,19 & 160 & 15,1 & 84 & 16 & 391 & 14,5 & 152 & 11,36 & 290 & 8,44 \\
\hline 0 a 34 & 104 & 43,15 & 164 & 27,1 & 255 & 28,27 & 369 & 34,84 & 201 & 38,21 & 994 & 37 & 393 & 29,37 & 810 & 23,6 \\
\hline a 49 & 51 & 21,16 & 122 & 20,1 & 156 & 17,29 & 181 & 17,09 & 105 & 20 & 512 & 19,04 & 199 & 14,87 & 364 & 10,6 \\
\hline a 64 & 14 & 5,8 & 42 & 7 & 65 & 7,2 & 95 & 8,97 & 40 & 7,6 & 208 & 7,73 & 103 & 7,69 & 178 & 5,18 \\
\hline 65 a 79 & 7 & 2,9 & 15 & 2,41 & 22 & 2,43 & 25 & 2,36 & 16 & 3,04 & 85 & 3,17 & 26 & 1,94 & 58 & 1,68 \\
\hline $80 a+$ & 0 & & 4 & 0,66 & 1 & 0,11 & 7 & 0,66 & 0 & 99,48 & 12 & 0,44 & 5 & 0,37 & 3 & 0,08 \\
\hline Totol & 211 & $00 \%$ & 605 & $1000 \%$ & 902 & 1000 & & 1000 & 526 & & & & & & & \\
\hline
\end{tabular}

Fonte: Elaborado pelos autores 
mostraram que a integração entre os dois programas é viável e representa otimização de recursos ao evitar a duplicidade das visitas e possibilita um maior envolvimento da comunidade no controle do dengue.

Segundo Gonçalves Neto e Rebêlo (2004), em São Luís, as dificuldades também são operacionais: aumento do número de imóveis em consequência da ocupação desordenada; capacitação inadequada do pessoal de campo; dificuldades de operacionalização na execução das ações de campo; quebra no processo de rotina das supervisões; frequente rotatividade dos convênios; interrupção constante do trabaIho do ultrabaixo volume (UBV); indisponibilidade de recursos orçamentários e financeiros; grande número de pendências por causa de imóveis fechados; dificuldade de manter a articulação com outros segmentos institucionais; dificuldade de estabelecer parceria de serviço com os municípios limítrofes; e atraso no repasse para a Secretaria Municipal de Saúde do Município de São Luís do insumo cipermetrina, para tratamento peri focal em pontos estratégicos. Em estudo sobre a epidemiologia da dengue no município de Campinas, São Paulo em 1998, por meio de inquérito populacional pós-epidêmico, Lima et al. (2007) concluíram que a prevalência de anticorpos para dengue na população de Campinas mostrou-se mais baixa que a encontrada em outros inquéritos realizados no país, em cidades médias e grandes.

Ao avaliar as ações de educação em saúde para prevenção e controle da dengue em Icaraí (Caucaia - Ceará), Sales (2008), concluiu que as epidemias de dengue são responsáveis no mundo, anualmente, por milhares de casos e óbitos e, no Brasil, o nível endêmico dessa doença está relacionado à elevada infestação domiciliar pelo Aedes aegypti e infestações humanas pelos diferentes sorotipos do vetor.

Em relação ao sexo de prevalência dos casos de dengue constatou-se que as pessoas do sexo feminino foram mais acometidas pela doença do que os pacientes do sexo masculino no período de 2000 a 2007, com índice de ocorrência significativo. O mesmo resultado foi encontrado por Sales (2007), em estudo avaliativo das ações de educação em saúde para prevenção e controle da dengue em Icaraí (Caucaia - Ceará), que em relação ao sexo encontrou a maior frequência de casos da doença entre as mulheres com $69 \%$ das ocorrências.

No que se refere à faixa etária de prevalência nos casos notificados de dengue encontrou-se neste estudo a maior frequência para a faixa etária dos 20 aos 34 anos entre 2000 a 2007. Este resultado está em consonância com o obtido por Sales (2007) em seu estudo onde a faixa etária predominante com 56\% dos casos foi de 21 a 40 anos de idade. Resultado semelhante foi encontrado pelo Instituto Brasileiro de Geografia e Estatística (2008) nas cidades do Rio Grande do Nortes: Natal, Parnamirim, Nova Cruz, Caicó, Ceará Mirim,
Jaçanã, Macaíba, Monte Alegre, Santa Cruz e Parelha, onde a faixa etária que mais tem sido acometida pela dengue é dos 20 aos 49 anos. No entanto, as crianças têm sido mais facilmente acometidas pela dengue hemorrágica.

Estudo realizado por Vasconcelos et al. (1999) concluíram que a ocorrência de dengue na ilha de São Luís não foi uniforme no tocante à faixa etária, aumentando gradualmente com a idade. Desta forma, torna-se necessário o incremento da investigação em epidemiologia de pesquisa já constituídos, além do incentivo à pesquisa operacional para responder de forma ágil questões específicas dos programas de controle e avaliar o impacto destas ações. Para Gonçalves Neto e Rebêlo (2004), é de fundamental importância manter uma contínua atividade educativa de esclarecimento à população sobre o risco de proliferação doméstica do vetor.

Segundo Chiaravalloti Neto (2007), o controle da dengue não pode ser dissociado das políticas sociais de educação, saneamento, habitação e manutenção do espaço público, sendo necessários investimentos na estrutura dos serviços públicos para que ocorra a integração intersetorial e sejam criadas condições para que os agentes atuem sobre os problemas do meio ambiente.

\section{CONSIDERAÇÕES FINAIS}

A situação da dengue no Brasil tem sido uma preocupação constante para as autoridades sanitaristas. Esta realidade não tem sido diferente no município de São Luís que possui características propícias para ser uma zona endêmica da dengue e apresenta fatores climáticos favoráveis para a proliferação da doença. Além disso, os casos notificados nos últimos anos têm apresentado um aumento significativo entre a população ludovicense. Deste modo, pode-se perceber que a situação epidemiológica da dengue no município de São Luís ainda constitui um problema de saúde pública, sendo necessário um maior incremento para o controle e combate aos focos da doença, bem como a melhoria de programas de educação em saúde que sensibilize a população para a efetiva erradicação dos focos do vetor devido aos grandes criadouros domésticos.

Constatou-se que São Luís permanece uma região de contínuas reincidências de casos por dengue com número crescente de casos notificados anualmente, o que merece a atenção e esforço das autoridades sanitárias e população em geral para a solução da problemática local. Os programas de educação em saúde devem ser ampliados e revitalizados a fim de garantir uma maior abrangência no fornecimento de informações adequadas para a população no combate à dengue, também há a necessidade de um sistema eficiente de vigilância vetorial e epidemiológica para as doenças transmitidas por artrópodes bem 
como uma maior sensibilização para a erradicação do vetor.

\section{REFERÊNCIAS}

ARAÚJO, R. R.; NUNES, J. S. A. Relações geográficas entre o clima e a incidência de dengue na cidade de São Luís-Ma. Ciências Humanas em Revista, São Luís, v. 3, n.2, dez. 2005.

BRAGA, I. A; VALLE, D. Aedes aegypti: histórico do controle no Brasil. Epidemiologia e Serviços de Saúde, Brasília, DF, v. 16, n. 2, p. 113-118.

BRASIL. Ministério da Saúde. Fundação Nacional de Saúde. Departamento de Operações. Coordenação de Controle de Doenças Transmitidas por Vetores. Manual de dengue: vigilância epidemiológica e atenção ao doente. 2.ed. Brasília, DF, 1996.

BRASIL. Ministério da Saúde. Secretaria de Vigilância em Saúde. Relatório do seminário internacional de avaliação do PNCD. Brasília, DF, 2007.

CÂMARA, F. P. et al. Estudo retrospectivo (histórico) da dengue no Brasil:

características regionais e dinâmicas. Revista da Sociedade Brasileira de Medicina Tropical, v. 40, n. 2, p.192-196, mar./abr. 2007.

COSTA, F. F. G. et al. Estudo químicobiológico dos óleos essenciais de Hyptis martiusii, Lippia sidoides e Syzigium aromaticum frente às larvas do aedes aegypti. Revista Brasileira de Farmacognosia, v.15, n. 4, p. 304-309, out./dez. 2005.

COSTA, A. E. A.; FERREIRA, L. G.

Considerações sobre o dengue clássico e o Hemorrágico. Pharmacia Brasileira, p.49-54, jan./mar. 2002.

CHIARAVALLOTI NETO, F. O programa de controle do dengue em São José do Rio Preto, São Paulo, Brasil: dificuldades para a atuação dos agentes e adesão da população. Cad. Saúde Pública, Rio de Janeiro, v.23, n. 7, p.1656-1664, jul. 2007.

DONALÍSIO, M. R.; GLASSER, C. M. Vigilância entomológica e controle de vetores do dengue. Rev. Bras. Epidemiol., v. 5, n.3, p. 259-272, 2002.

DUARTE, H. H. P.; FRANÇA, E. B. Qualidade dos dados da vigilância epidemiológica da dengue em Belo Horizonte, MG. Revista de Saúde Pública, v. 40, n. 1, p. 134-142, 2006. FOCACCIA, R. V. Tratado de infectologia. 3.ed. São Paulo: Atheneu, 2006.
GONÇALVES NETO, V. S.; REBÊLO, J. M. $M$. Aspectos epidemiológicos do dengue no Município de São Luís, Maranhão, Brasil, 1997-2002. Cad. Saúde Pública, Rio de Janeiro, v. 20, n.5, p. 1424-1431, set./ out.2004.

INSTITUTO BRASILEIRO DE GEOGRAFIA E ESTATÍSTICA (IBGE), Área Territorial Oficial, Resolução no 5 de 10 de outubro de 2002. Disponível em: <http://www.ibge.gov.br/ home/geociencias/areaterritorial/resolucao. shtm>. Acesso em: 13 out. 2008.

. Evolução da divisão territorial do

Brasil 1872-2010. 2011. Disponível em: <ftp://geoftp.ibge.gov.br/organizacao_ territorial/divisao_territorial/evolucao_da_ divisao_territorial_do_brasil_1872_2010/ evolucao_da_divisao_territorial_do_brasil_ publicacao_completa.pdf>. Acesso em: 22 ago. 2011.

LIMA, V. L. C. et al. Dengue: inquérito populacional para pesquisa de anticorpos e vigilância virológica no Município de Campinas, São Paulo, Brasil. Cad. Saúde Pública, Rio de Janeiro, v.23, n.3, p.669-680, mar. 2007.

MACIEL, I. J.; SIQUEIRA JR, J. B.; MARTELLI, C. M. T. Epidemiologia e desafios no controle do dengue. Revista de Patologia Tropical, Goiânia, v. 37, n. 2, p. 111-130, 2008.

MEDRONHO, R. A. Dengue e o ambiente urbano. Rev. Bras. Epidemiol., v. 2, n. 9, p.159-161, 2006.

SÃo LUÍS. Secretaria Municipal de Saúde. Relatório anual de atividades, período: 2002. São Luís: Secretaria Municipal de Saúde, 2003.

Relatório quadrienal de atividades, período: 1997 a 2000. São Luís: Secretaria Municipal de Saúde, 2001.

SALES, F. M. S. Ações de educação em saúde para prevenção e controle da dengue: um estudo em Icaraí, Caucaia, Ceará. Ciênc. saúde coletiva, Rio de Janeiro, v. 13, n. 1, 2008.

SINGHI, S.; KISSOON, N.; BANSAL, A. Dengue e dengue hemorrágico: aspectos do manejo na unidade de terapia intensiva. $J$ Pediatr., Rio de Janeiro, v. 2, n. 83, p. 2235, 2007.

TEIXEIRA, M. G. et al. Avaliação de impacto de ações de combate ao Aedes aegypti na cidade de Salvador, Bahia. Rev. Bras. Epidemiol., v. 5, n. 1, p. 108-115. 2002. 
TOLEDO, A. L. A. et al. Confiabilidade do diagnóstico final de dengue na epidemia 20012002 no Município do Rio de Janeiro, Brasil.

Cad. Saúde Pública, Rio de Janeiro, v. 22, n.5, p. 933-940, maio 2006.

TORRES, E. M. Dengue. Rio de Janeiro: Fiocruz; 2005.
VABO, K. A. Achados ultrassonográficos abdominais em pacientes com dengue. Radiol. Bras. 2004; v. 37, n. 3, p. $159-162$.

VASCONCELOS P.F.C. et al. Inquérito soroepidemiológico na Ilha de São Luís durante epidemia de dengue no Maranhão. Rev Soc Bras Med Trop. 1999, v. 32, p. 171-179. 